\title{
Diffusion-Weighted Imaging with Dual-Echo Echo-Planar Imaging for Better Sensitivity to Acute Stroke
}

\author{
S.J. Holdsworth, K.W. Yeom, M.U. Antonucci, J.B. Andre, J. Rosenberg, M. Aksoy, M. Straka, N.J. Fischbein,
}

R. Bammer, M.E. Moseley, G. Zaharchuk, and S. Skare

\begin{abstract}
BACKGROUND AND PURPOSE: Parallel imaging facilitates the acquisition of echo-planar images with a reduced TE, enabling the incorporation of an additional image at a later TE. Here we investigated the use of a parallel imaging-enhanced dual-echo EPI sequence to improve lesion conspicuity in diffusion-weighted imaging.
\end{abstract}

MATERIALS AND METHODS: Parallel imaging-enhanced dual-echo DWI data were acquired in 50 consecutive patients suspected of stroke at 1.5T. The dual-echo acquisition included 2 EPI for 1 diffusion-preparation period (echo 1 [TE $=48 \mathrm{~ms}$ ] and echo 2 [TE $=105 \mathrm{~ms}$ ]). Three neuroradiologists independently reviewed the 2 echoes by using the routine DWI of our institution as a reference. Images were graded on lesion conspicuity, diagnostic confidence, and image quality. The apparent diffusion coefficient map from echo 1 was used to validate the presence of acute infarction. Relaxivity maps calculated from the 2 echoes were evaluated for potential complementary information.

RESULTS: Echo 1 and 2 DWIs were rated as better than the reference DWI. While echo 1 had better image quality overall, echo 2 was unanimously favored over both echo 1 and the reference DWI for its high sensitivity in detecting acute infarcts.

CONCLUSIONS: Parallel imaging-enhanced dual-echo diffusion-weighted EPI is a useful method for evaluating lesions with reduced diffusivity. The long TE of echo 2 produced DWIs that exhibited superior lesion conspicuity compared with images acquired at a shorter TE. Echo 1 provided higher SNR ADC maps for specificity to acute infarction. The relaxivity maps may serve to complement information regarding blood products and mineralization.

ABBREVIATIONS: DW = diffusion-weighted; GRAPPA = generalized autocalibrating partially parallel acquisition; GRE = gradiant-recalled echo; PI = parallel imaging; $R_{2}=$ relaxivity map

$D$ iffusion-weighted imaging plays a key role in evaluating multiple neurologic diseases, including stroke. ${ }^{1-7}$ In DWI, the image intensity reflects the rate of water diffusion at a given location, but DWI also remains sensitive to $\mathrm{T} 1$ and $\mathrm{T} 2$ relaxation (and proton attenuation). T1 relaxation effects can be mitigated by using a long TR to allow longitudinal relaxation. Due to the insertion of the diffusion gradients and the associated TE prolonga-

Received August 9, 2013; accepted after revision December 5.

From the Department of Radiology (S.J.H., K.W.Y., M.U.A., J.R., M.A., M.S., N.J.F., R.B., M.E.M., G.Z.), Stanford University, Stanford, California; Department of Radiology (J.B.A.), University of Washington, Seattle, Washington; and Clinical Neuroscience (S.S.), Karolinska Institute, Stockholm, Sweden.

This work was supported by the National Institutes of Health (2R01 NS047607-06, 2R01 EB00271108-A1, 5RO1 EB008706, 5R01 EB01165402-02), the Center of Advanced MR Technology at Stanford (P41 EB015891), and the Lucas Foundation.

Please address correspondence to Samantha Holdsworth, PhD, Department of Radiology, Stanford University, Lucas Center, 1201 Welch Rd, Stanford, CA 943055488; e-mail: sholdsworth@stanford.edu

- Indicates open access to non-subscribers at www.ajnr.org

http://dx.doi.org/10.3174/ajnr.A3921 tion, however, T2 effects cannot be mitigated during image acquisition. Thus, T2 and diffusion effects are entangled on DWI, though it is often clinically important to differentiate their relative contribution to the final image. Therefore, the quantitative ADC images are produced, which remove the T2 weighting effect. ${ }^{8,9}$

The most efficient, reliable, and thereby conventional method for DWI acquisition is with the EPI technique. A significant limitation of diffusion-weighted (DW)-EPI is the presence of susceptibility artifacts, which manifest as geometric distortion, signal drop-out, and image blurring. To reduce such artifacts in DWEPI, parallel imaging (PI) ${ }^{10,11}$ can be used to accelerate EPI. ${ }^{12,13}$ In PI-enhanced EPI, the echo train is shortened, thereby reducing TE. A short TE in DWI is typically considered as advantageous because it results in a higher SNR and reduces the T2 contrast. However, because many lesions with reduced diffusivity also have prolonged T2 values compared with the surrounding tissue, ${ }^{14}$ a short TE may reduce the conspicuity of ischemic lesions.

With the hypothesis that improved conspicuity of lesions with reduced diffusivity can be achieved by longer TEs than in conven- 


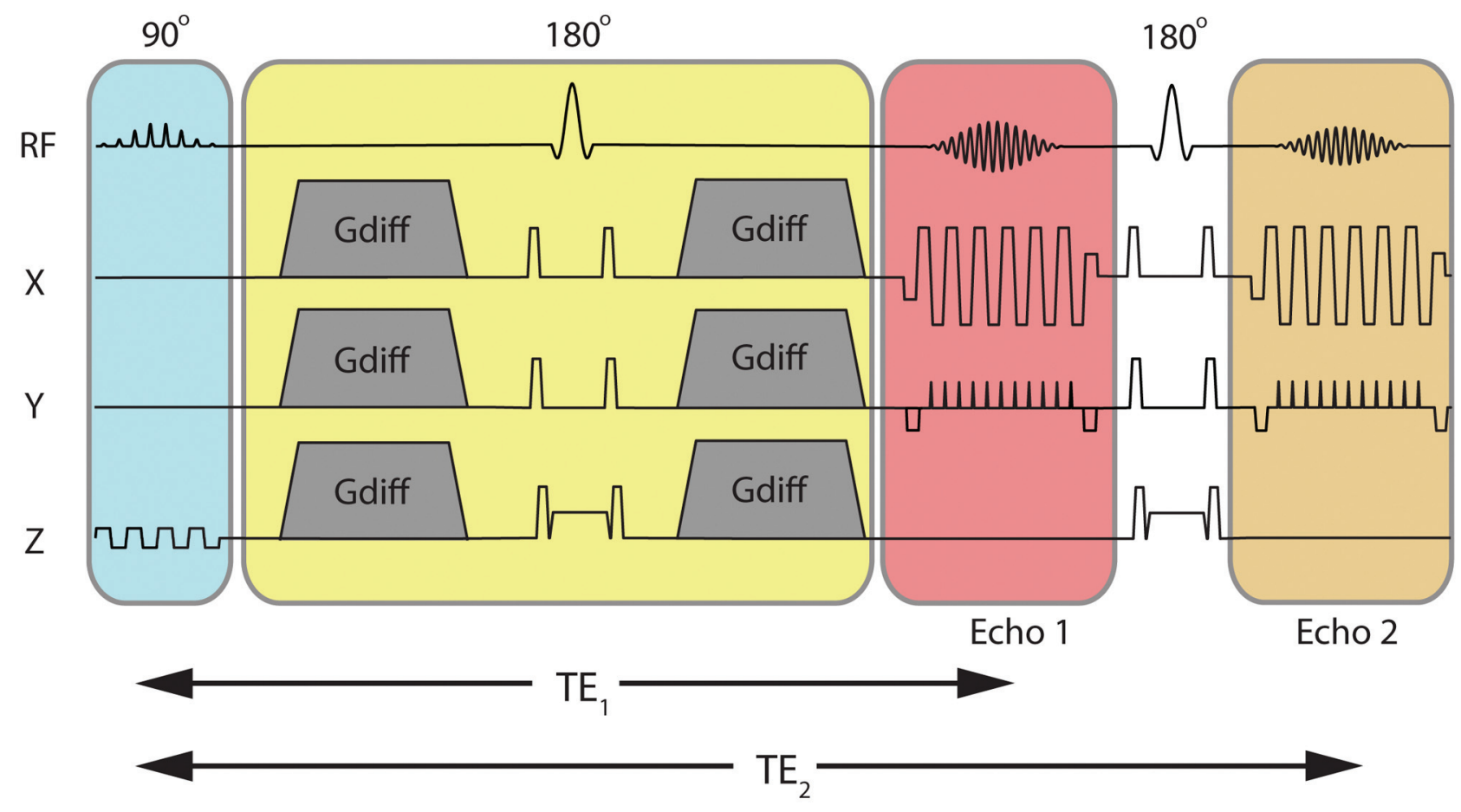

FIG 1. Dual-echo DWI pulse sequence showing the section-select module (blue); diffusion preparation period (yellow); and the first and second imaging echoes (echoes 1 and 2 acquired at 2 different $T E s, T E_{1}$ and $T E_{2}$ ) separated by a $180^{\circ}$ refocusing pulse.

tional practice, 1 option is to simply increase the TE (within reasonable limits) of a DWI acquisition. Another option is to increase the b-value (which also increases the contribution of reduced diffusion in the signal). Unfortunately, both approaches reduce the SNR of the ADC image (computed from the $b=0$ and the DWI), which is used for diagnostic specificity. Another option is to add a second DW echo within the same $\mathrm{TR}^{15,16}$ - an approach that does not significantly impact the overall scanning time. Figure 1 depicts this dual-echo DWI approach, with 2 EPI trajectories acquired for 1 diffusion-preparation period (echo 1 and echo 2 , respectively). Here, echo 2 is used to increase the sensitivity to lesions, and echo 1 provides a higher SNR image used to calculate the ADC. Furthermore, relaxivity $\left(R_{2}\right)$ maps can be calculated from the 2 $b=0$ images to potentially reveal an additional source of image contrast without additional scanning time.

In this work, we imaged patients clinically suspected of acute or subacute stroke by using a PI-enhanced dual-echo DW-EPI sequence on a $1.5 \mathrm{~T}$ clinical scanner to explore the impact that a longer TE might have on clinical management and patient outcome. Our aim was to evaluate the 2 diffusion-weighted echoes (echo 1 and echo 2) obtained from the dual-echo sequence on image quality, lesion quality, and diagnostic quality metrics, and to evaluate relaxivity maps calculated from the 2 echo images as a potential additional source of image contrast.

\section{MATERIALS AND METHODS \\ Patient Studies}

All data were acquired on an inpatient 1.5T MR imaging system (Signa HD; GE Healthcare, Milwaukee, Wisconsin) equipped with an 8-channel head coil. With institutional review board approval, 50 consecutive adult patients who were clinically suspected of stroke were scanned with the dual-echo DWI sequence
Table 1: Clinical history of the $\mathbf{5 0}$ patients who were suspected of stroke and scanned for this study

\begin{tabular}{lcc}
\hline \multicolumn{1}{c}{ Clinical History } & $\begin{array}{c}\text { No. of } \\
\text { Patients }\end{array}$ & $\begin{array}{c}\text { No. of Patients } \\
\text { with Lesions with } \\
\text { Reduced Diffusivity }\end{array}$ \\
\hline Moyamoya disease & 5 & 5 \\
Transient ischemic attack & 16 & 5 \\
Stroke & 14 & 14 \\
Cavernous malformation & 3 & 2 \\
Hemorrhage & 5 & 5 \\
Vasospasm post-subarachnoid & 3 & 3 \\
$\quad$ hemorrhage & 1 & 0 \\
Metabolic disease & 2 & 1 \\
Brain tumor & 1 & 1 \\
Headache & 50 & 36 \\
Total No. of patients & & \\
\hline
\end{tabular}

${ }^{a}$ The number of patients with lesions with reduced diffusivity present on $\geq 1$ of the scanned DWI sequences is also shown.

between January 13, 2011, and April 6, 2011. The product DWI sequence used as part of standard of care at our institution was used as the criterion standard comparison DWI sequence for this study. We also acquired additional routine MR images relevant to the clinical scenario: T2-weighted FLAIR, T2-weighted gradientrecalled echo (GRE), fast spin-echo, MRA of the circle of Willis, and bolus perfusion imaging. The final clinical diagnosis was based on the imaging findings that were correlated with appropriate clinical symptoms (Table 1 ).

\section{Imaging Sequence and Reconstruction}

Our generalized autocalibrating partially parallel acquisition (GRAPPA)-accelerated DWI sequence was modified to include a second-echo EPI trajectory succeeding a second $180^{\circ}$ refocusing pulse (Fig 1). Patient data were acquired by using the following imaging parameters: GRAPPA acceleration factor of $\mathrm{R}=3, \mathrm{FOV}=$ 
Table 2: Agreement among readers on specific ratings using a weighted $\kappa$ statistic $(N=50)^{a}$

\begin{tabular}{lccccccc}
\hline & \multicolumn{3}{c}{ Echo 1 } & & \multicolumn{3}{c}{ Echo 2 } \\
\cline { 2 - 4 } & $\begin{array}{c}\text { Diag } \\
\text { Conf }\end{array}$ & Conspicuity & Quality & & Conf & Conspicuity & Quality \\
\hline A vs B & -0.05 & 0.10 & 0.00 & 0.07 & -0.00 & -0.09 \\
A vs C & 0.10 & 0.13 & 0.31 & 0.16 & 0.13 & -0.09 \\
B vs C & 0.02 & -0.02 & 0.00 & 0.09 & 0.14 & 0.31 \\
\hline
\end{tabular}

Note:-Diag Conf indicates diagnostic confidence.

${ }^{\text {a }}$ All ratings are with $P>.14$.

Table 3: Preference for the second echo over the first echo

\begin{tabular}{ccccc}
\hline Reader & Fraction & Percentage & $95 \%$ CI & $\boldsymbol{P}$ Value (1-tailed) \\
\hline Reader A & $35 / 50$ & $70 \%$ & $(55 \%-82 \%)$ & .003 \\
Reader B & $38 / 50$ & $76 \%$ & $(62 \%-87 \%)$ & $<.001$ \\
Reader C & $34 / 50$ & $68 \%$ & $(53 \%-80 \%)$ & .008 \\
\hline
\end{tabular}

Table 4: Percentage of cases rated greater than the product DWI $(N=50)$

\begin{tabular}{|c|c|c|c|c|c|c|}
\hline & \multicolumn{3}{|c|}{ Echo 1} & \multicolumn{3}{|c|}{ Echo 2} \\
\hline & Diag & & & Diag & & \\
\hline & Conf & Conspicuity & Quality & Conf & Conspicuity & Quality \\
\hline$A$ vs $B$ & $92 \%$ & $84 \%$ & $100 \%$ & $96 \%$ & $98 \%$ & $100 \%$ \\
\hline$A$ vs $C$ & $78 \%$ & $66 \%$ & $100 \%$ & $96 \%$ & $98 \%$ & $92 \%$ \\
\hline$B$ vs $C$ & $96 \%$ & $92 \%$ & $100 \%$ & $96 \%$ & $94 \%$ & $94 \%$ \\
\hline
\end{tabular}

Note:-Diag Conf indicates diagnostic confidence.

Table 5: Mean (SD) of ratings $(N=50)$

\begin{tabular}{|c|c|c|c|c|c|c|}
\hline & \multicolumn{2}{|c|}{$\begin{array}{l}\text { Diagnostic } \\
\text { Confidence }\end{array}$} & \multicolumn{2}{|c|}{ Conspicuity } & \multicolumn{2}{|c|}{ Quality } \\
\hline & Echo 1 & Echo 2 & Echo 1 & Echo 2 & Echo 1 & Echo 2 \\
\hline Reader A & $5.7(0.9)$ & $6.4(0.7)$ & $5.1(1.0)$ & $6.3(0.9)$ & $6.3(0.5)$ & $5.5(0.6)$ \\
\hline Reader B & $4.8(0.7)$ & $5.6(0.6)$ & $4.4(0.9)$ & $6.0(0.7)$ & $5.8(0.4)$ & $4.5(1.0)^{a}$ \\
\hline Reader C & $5.8(0.6)$ & $6.4(0.8)$ & $5.2(0.7)$ & $6.1(0.9)$ & $6.1(0.3)$ & $4.8(0.7)^{b}$ \\
\hline
\end{tabular}

$24 \mathrm{~cm}$, acquisition matrix $=192 \times 192,5-\mathrm{mm} / 0$-mm section thickness/gap, partial Fourier encoding with 24 overscans, $\mathrm{TE}_{1} / \mathrm{TE}_{2}=$ 48/105 ms, Stejskal-Tanner diffusion preparation, ${ }^{17}$ tetrahedral encoding (4 diffusion directions) with $b=1000 \mathrm{~s} / \mathrm{mm}^{2}$, and $1 \mathrm{~T} 2-$ weighted $(b=0)$ image. Each volume was acquired by using 3 interleaves. The fully sampled $b=0$ image was formed after combining the 3 interleaves and was used to enable the estimation of GRAPPA weights. These estimated GRAPPA weights were applied to all interleaves of all acquired volumes separately, including the $b=0$ volume itself. ${ }^{13}$ A TR of 3 seconds was used to keep T1 saturation effects small in the brain parenchyma (resulting in $95.8 \%$ and $99.3 \% \mathrm{~T} 1$ recovery at $1.5 \mathrm{~T}$, assuming a $\mathrm{T} 1=950 \mathrm{~ms} / 600 \mathrm{~ms}$ for gray and white matter, respectively ${ }^{18}$ ). A maximum of 23 sections was permitted in a TR of 3 seconds, and the total scanning time was 2 minutes 15 seconds. The product DWI sequence scanned routinely at our institution was used as the criterion standard DWI sequence for this study, which used a PI-enhanced (sensitivity encoding-type) EPI sequence with the following parameters: matrix size $=128 \times 128$, acceleration factor $\mathrm{R}=$ 2, partial Fourier encoding, TR/TE $=8$ seconds $/ 70 \mathrm{~ms}$, twice-refocused $^{19,20}$ diffusion preparation, 23 sections with a $5-\mathrm{mm} / 0-\mathrm{mm}$ section thickness/gap, partial Fourier encoding with 16 overscans, 3 diffusion directions with $b=1000 \mathrm{~s} / \mathrm{mm}^{2}$, one T2-weighted $(b=0)$ image, and a scanning time of 50 seconds.

The postprocessing of the dual-echo DWI data was performed
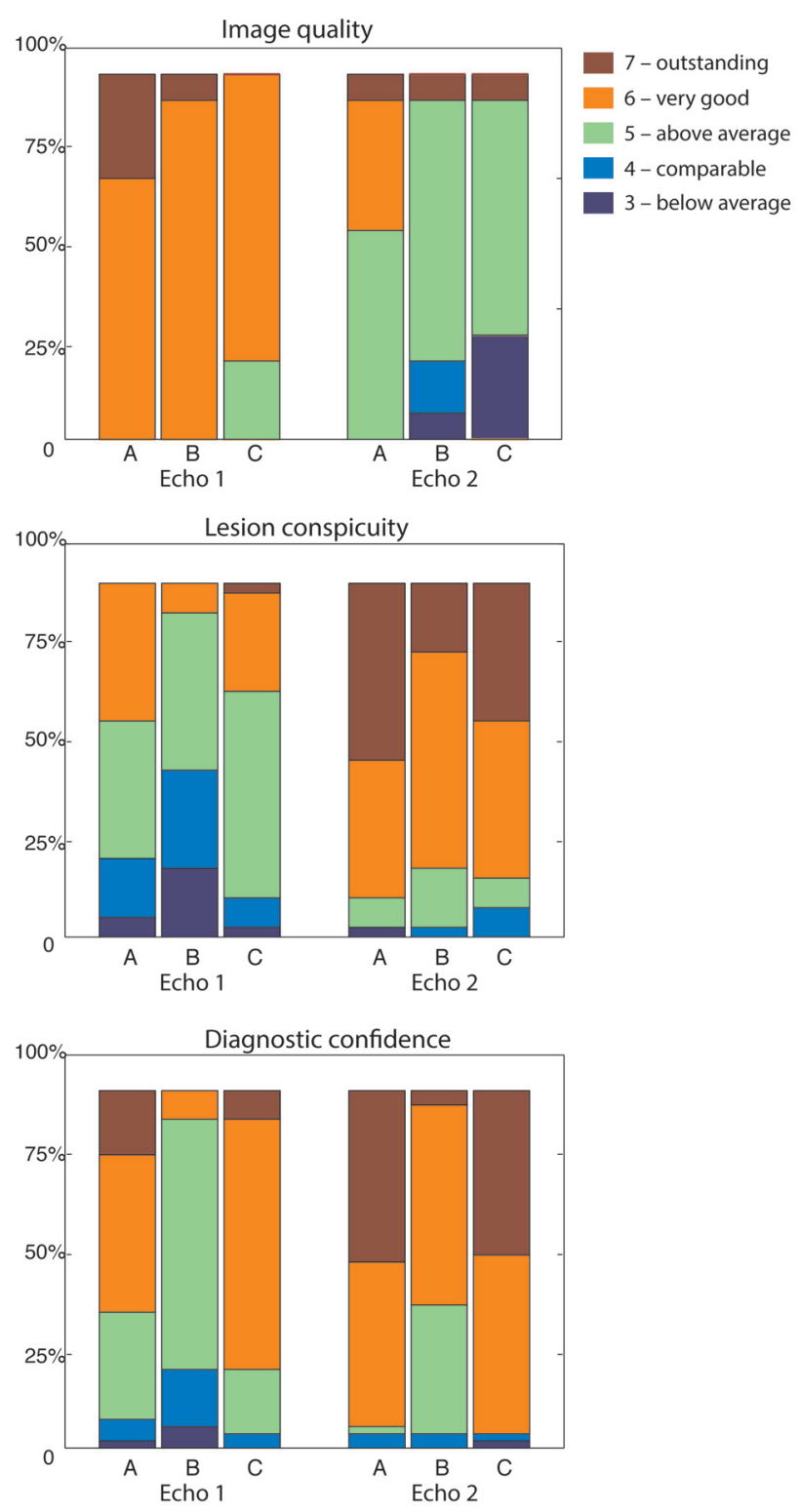

FIG 2. Assessment of readers $A, B$, and $C$ of image quality, lesion conspicuity, and diagnostic confidence. Nominal values of 3-7 represent reader grading compared with the product DW-EPI (assigned as 4), represented by the percentage of total assigned values. Note that values of 1-2 (corresponding to "nondiagnostic" and "poor") are not shown because these values were not assigned in this study.

by using a compiled and multithreaded Matlab code (Version 7.8.0; MathWorks, Natick, Massachusetts). Both echoes (echo 1 and echo 2) were reconstructed and sent to our hospital image data base (PACS). These DWI datasets included the isotropic DWI from the 2 echoes $\left(E_{1}\right.$ and $\left.E_{2}\right)$; the isotropic ADC calculated from the $b=0$ and $b=1000 \mathrm{~s} / \mathrm{mm}^{2}$ from echo 1 ; and the relaxivity maps, $R_{2}$, calculated from the average of the $b=0$ and $b=$ $1000 \mathrm{~s} / \mathrm{mm}^{2}$ relaxivity maps as follows:

$$
R 2=\frac{1}{2}\left(R_{E 1}+R_{E 2}\right)=\frac{1}{2}\left(\frac{\log \left(B 0_{2} / B 0_{1}\right)}{T E_{2}-T E_{1}}+\frac{\log \left(D W_{2} / D W_{1}\right)}{T E_{2}-T E_{1}}\right),
$$

where $B 0$ represents the $b=0 \mathrm{~s} / \mathrm{mm}^{2}$ image, and $D W$, the $b=$ $1000 \mathrm{~s} / \mathrm{mm}^{2}$ image at 2 TEs. 


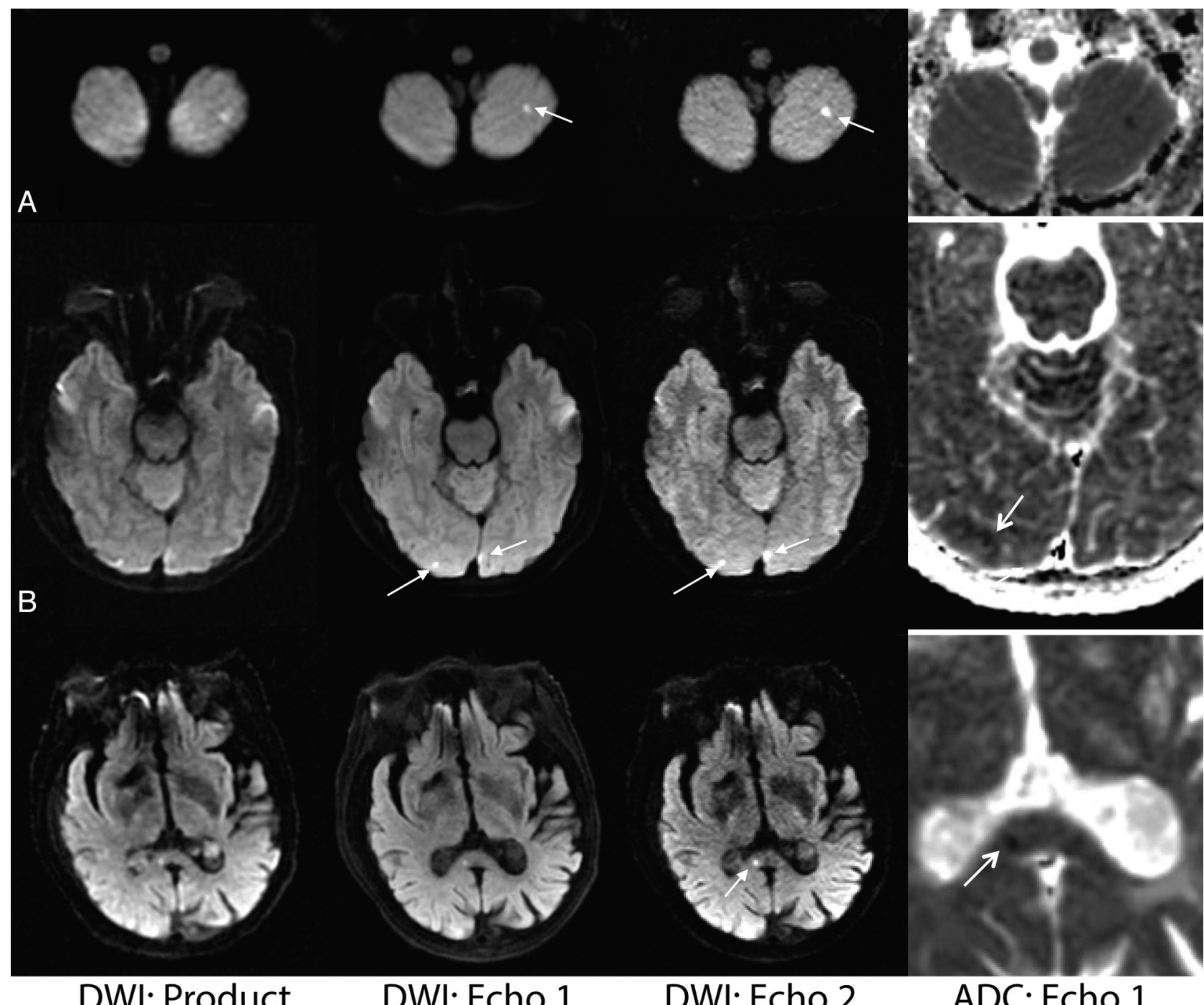

FIG 3. Comparison of the vendor-supplied (product) DWI, echo 1 , and echo $2 \mathrm{DWI}$ acquired from the dual-echo sequence on 3 patients (from left to right). The ADC (calculated from echo 1) shows the presence of reduced diffusivity in each lesion (far right column). $A$, A 62-year-old female patient with stroke. B, A 49-year-old male patient with vasospasm and infarction post-aneurysm clipping. C, An 88-year-old woman presenting with strokelike symptoms. Note that the small infarct in the splenium of the corpus callosum present on the dual-echo DWIs was initially missed on the product DWI.

\section{Imaging Evaluation}

Three blinded board-certified neuroradiologists (readers A-C) with 7 years', 5 years', and 1 year experience, respectively, independently evaluated the DWIs of echo 1 and echo 2 for lesions with reduced diffusivity in 50 patients admitted for possible stroke. Each reader, blinded to the clinical symptoms and final diagnosis, first viewed the DWI from echo 1, followed by the DWI from echo 2, by using the reference product DWI as the criterion standard. The neuroradiologists made following evaluations:

1) Presence/absence of DWI-hyperintense lesions on echoes 1 and 2 (yes/no)

2) Diagnostic confidence for each echo DWI (echo 1 and 2) relative to the reference image

3) For cases with DWI-hyperintense lesions, the lesion conspicuity of each echo image relative to the reference image

4) For cases without DWI-hyperintense lesions, the image quality of each echo image relative to the reference image
5) Overall preference for the DWI from echo 1 or echo 2 (binary forced choice).

Diagnostic confidence, lesion conspicuity, and image quality for the DWI echo images were scored on the following 7-point Likert scale: 1 , nondiagnostic; 2 , poor; 3 , acceptable; 4 , comparable with product DWI (the criterion standard); 5 , above average; 6 , very good; and 7 , outstanding.

Reader B made the following additional evaluations:

1) The number of new lesions seen on the DWI of echo 2 compared with that of echo 1

2) The presence of something new on the DWI of echo 2 compared with that of echo 1 (yes/no)

3) Clinical impact: if additional information was garnered from the DWI of echo 2, would this alter clinical management, such as therapy or clinical referral? ( 0 , no; 1 , possible; 2 , yes). 

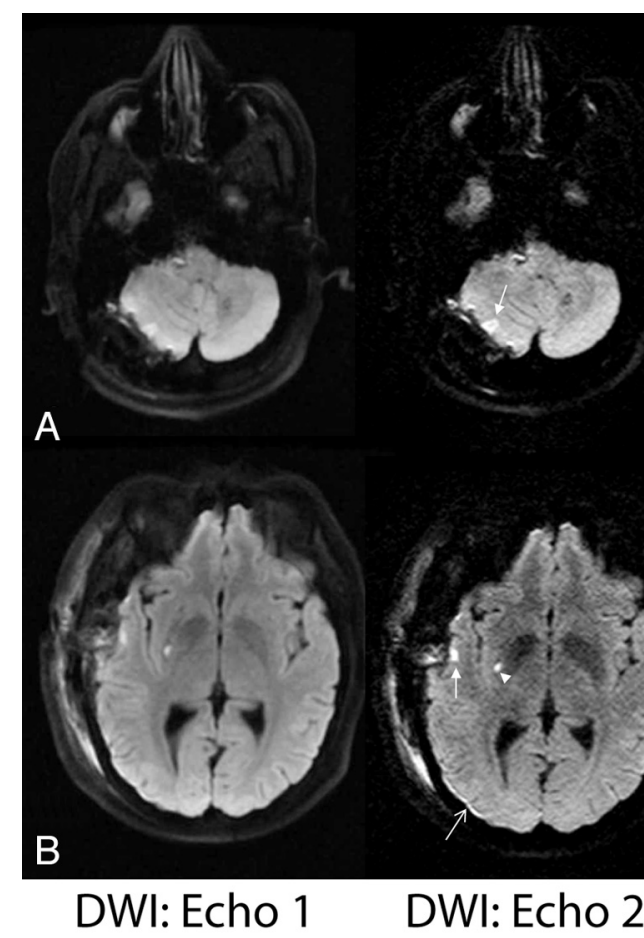

DWI: Echo 2
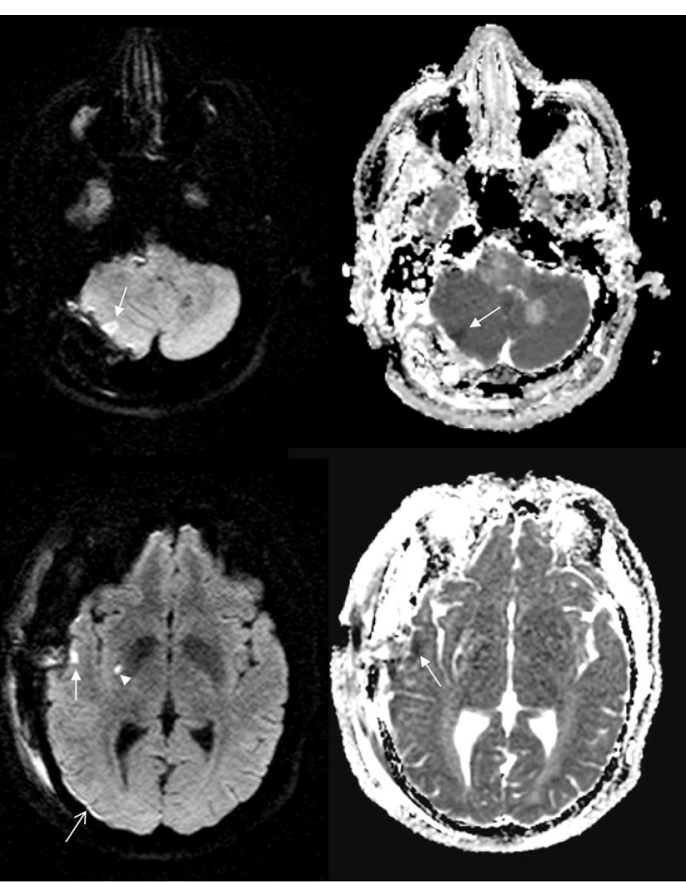

ADC: Echo 1

FIG 4. Examples of improved lesion detection of echo 2. A, A 56-year-old man status post posterior fossa tumor resection. Right cerebellar injury is more conspicuous on echo 2 and was confirmed to have reduced diffusion based on ADC. B, A 39-year-old man with known Moyamoya disease status post right superficial temporal artery to middle cerebral artery anastomosis presented with acute strokelike symptoms. Superficial temporal cortical lesion (closed arrow) and a punctate putaminal lesion (arrowhead) are confirmed with reduced diffusivity based on the ADC map. Also note improved delineation of a small subdural hematoma on echo 2 (open arrow).

For this last scenario, a representative case might be one in which echo 2 identified additional focal lesions in the same vascular territory. In such a case, clinical management would likely not be altered (score 0 ). However, if other lesions were seen in new vascular distribution, a more detailed clinical investigation may ensue for a potential embolic source (score 1). Alternatively, if echo 2 identified a new lesion in a case previously interpreted as having negative findings, management may alter in terms of patient risk stratification for future stroke or initiating therapies for the current lesion or for future stroke prevention (score 2).

Lesion conspicuity and diagnostic confidence took into account the readers' ability to readily observe lesions against the background tissue, as well as lesion delineation. Diagnostic confidence, clinical impression, and clinical impact were assessed in the context of other imaging data (ADC, FLAIR, GRE). Small lesions on echo 2 that were difficult to confirm on ADC were given independent assessments from the readers as to whether their presence increased or decreased diagnostic confidence. Final clinical diagnosis was made by using all clinical and imaging material (including previous examinations, if available) and served as ground truth for the study.

Lesions detected by echo 2 were validated for the presence of acute infarction by using the ADC from echo 1. If these lesions did not have reduced diffusivity, FLAIR images were used to identify possible associated parenchymal T2 hyperintensity. Extra-axial hemorrhage was excluded from this assessment.

\section{Relaxivity Maps}

The relaxivity maps were viewed and weighed against all other imaging data for potential complementary diagnostic information.

\section{Statistical Analysis}

All statistical analyses were performed with STATA, Release 11.1 (StataCorp, College Station, Texas). Agreement among readers was assessed by a linearly weighted $\kappa$ statistic. Preference for echo 1 or echo 2 was tested with an exact binomial test with a null value of 0.5 . Tests for superiority compared with standard imaging were performed with 2-tailed Wilcoxon signed rank tests with a null median of 4 on the Likert scale (comparable with product DWI). Tests for differences in ratings between echo 1 and echo 2 were performed by using 2-tailed Wilcoxon signed rank tests. There was no correction for multiple comparisons.

\section{RESULTS}

Table 1 shows the clinical diagnoses of all the patients who were referred for diagnostic work-up for stroke-like symptoms. Lesions with reduced diffusivity were found in 36 of the 50 patients. Readers $\mathrm{B}$ and $\mathrm{C}$ were in perfect agreement in assessing the presence of lesions with reduced diffusivity, and both differed from reader A in 4 cases ( 2 patients with lesions that had reduced diffusivity, and 2 lesions without reduced diffusivity; $\kappa=0.80$; $95 \%$ CI, 0.58-0.95).

There was little agreement among readers for specific ratings (Table 2), yet all 3 readers preferred the DWI of echo 2 over echo 1 for patients with lesions with reduced diffusivity (Table 3 ). Echo 1 and echo 2 images were consistently preferred over the product DWI (reference) image (Table 4). For all readers, echo 1 had better image quality than echo 2 because echo 1 was subjectively a more aesthetically pleasing and higher SNR image. However, echo 2 was rated higher than echo 1 for lesion conspicuity and diagnostic confidence (Table 5). Figure 2 depicts individual reader assessment of image quality, lesion conspicuity, and diagnostic confidence for echoes 1 and 2, respectively, as a percentage of the total assigned values per reader. The median values for echo 1 and 2 assessments for all patients were the following: 6 and 5, respectively, for image quality; and 5 and 6, respectively, for lesion conspicuity and diagnostic confidence.

Seventy-two new lesions were identified on echo 2 DWI (that were not seen on echo 1) in $46 \%$ of evaluated patients. Of these, 67 were confirmed as lesions with reduced diffusivity based on the ADC generated from echo 1 . Three lesions were too small to assess; 2 were deemed to represent edema associated with T2 prolongation based on ADC. The 72 newly identified lesions with reduced diffusivity on echo 2 were seen in 18 patients with acute 


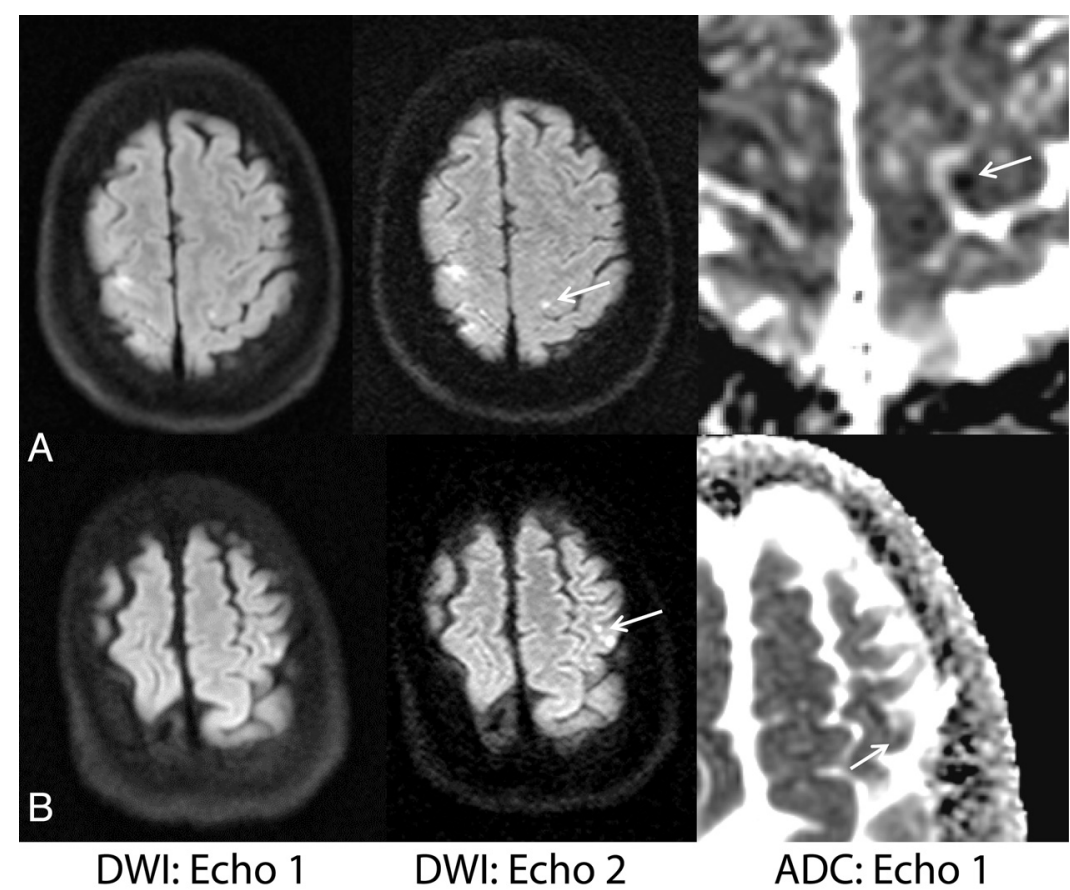

FIG 5. Examples in which additional lesions identified by echo 2 suggested a potential underlying mechanism of stroke and potentially altered diagnostic impression and clinical management. A, A 60-year-old woman with vasospasm after subarachnoid hemorrhage. B, A 65-yearold woman with embolic infarcts who also had lesions in the right cerebral hemisphere more inferiorly (not shown). On both patients, echo 2 showed missed sites of reduced diffusivity on the contralateral hemisphere (arrows), suggesting multiple vascular distribution involvement (vasospasm in multiple vascular territories or embolic source). Note that the lesions were retrospectively observed on echo 1 (but not on the product DWI). infarction and 3 patients with intracranial hemorrhage. Of the 18 patients with acute infarction, 2 had embolic infarcts from cardiac causes, 4 had infarcts related to underlying Moyamoya disease, 4 had ischemic lesions related to vasospasm and prior aneurysm coiling/clipping, 1 had ischemic changes adjacent to operative site, and 7 patients had infarcts that were not otherwise specified clinically. Due to its added sensitivity to diffusion lesions, it was predicted that echo 2 would have impacted stroke work-up in $16 \%$ of cases ( 8 patients) and potentially influenced $32 \%$ of cases ( 16 patients).

Figure 3 is representative of a case in which the routine product DWI sequence demonstrated lower diagnostic confidence compared with echoes 1 and 2 .

Figure 4 depicts 2 cases in which echo 2 demonstrates improved lesion conspicuity compared with echo 1 . These patients had recently undergone surgery, and the findings on echo 2-while not likely to alter clinical management as determined during the radiologists' readings-better defined the nature of the postoperative changes (such as local isch-

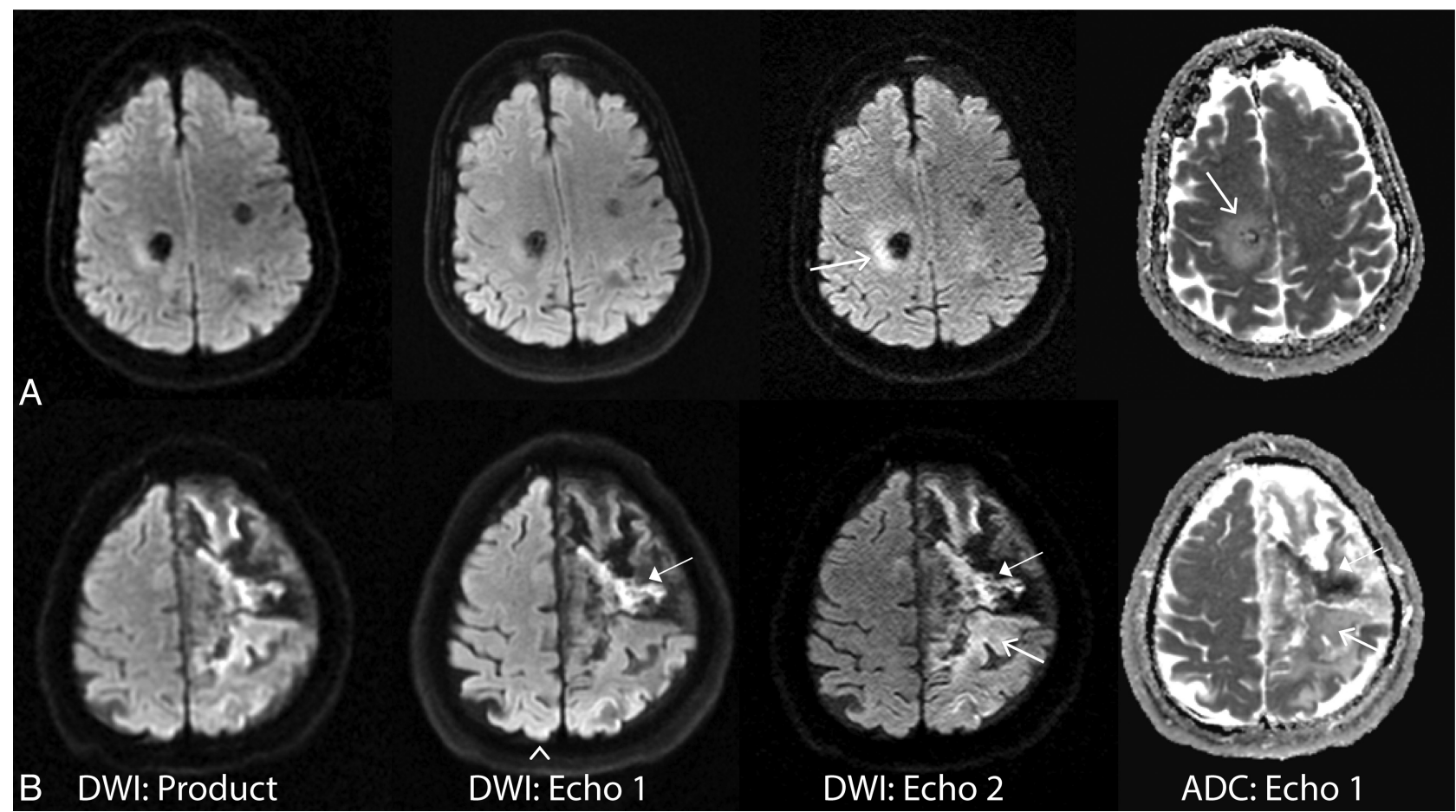

FIG 6. Examples in which the heightened sensitivity of echo 2 prompted further assessment by using ADC. A, A 61-year-old woman with hemorrhage from a cavernous malformation. Bright signal around the lesion was seen on echo 2 (and product) DWI and was confirmed as edema around a hemorrhage site (open arrows). B, A 69-year-old man with strokelike symptoms. Example of acute and subacute (closed arrows)/ chronic stroke (open arrows) on echo $2 \mathrm{DWI}$, also present on the product DWI. Also note the arrowhead on echo 1 showing unwanted heightened coil sensitivity in the posterior brain region. 


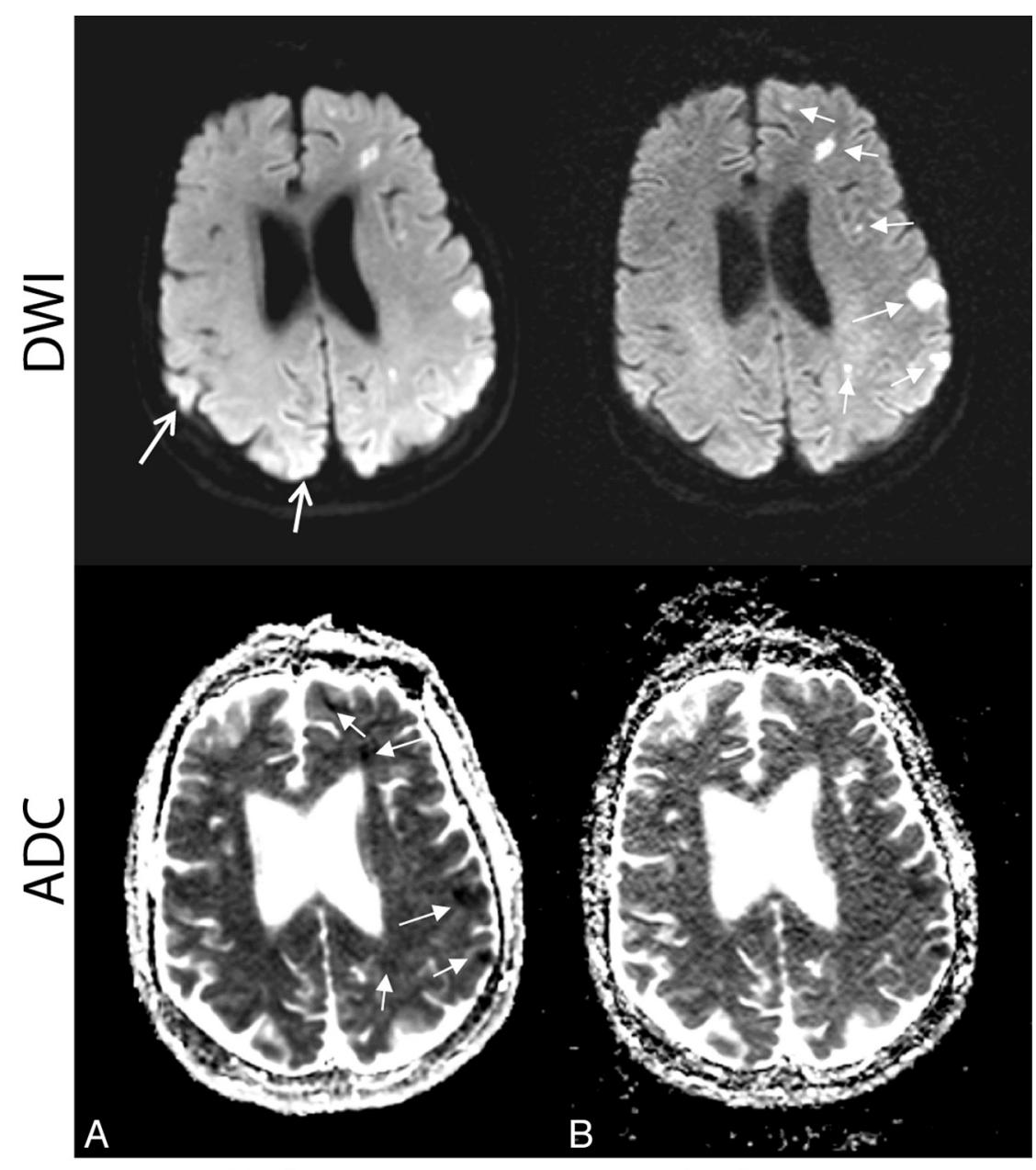

Echo 1

FIG 7. Case illustrating why echo 1 is more useful than echo 2 for calculating ADC maps used for the validation of acute infarct. Select images of a 66 -year-old woman with embolic infarcts. DWI and ADC maps for echo $1(A)$ and echo $2(B)$ are shown. The DWI of echo 2 was found to have much higher sensitivity to acute lesions (confirmed on the ADC of echo 1) than echo 1. However, because the ADC of echo 2 is plagued by noise, echo 1 is considerably more useful for calculating ADC maps used for the validation of acute stroke (closed white arrows). The open white arrows represent areas where it can be difficult to rule out stroke from heightened coil sensitivity in (particularly posterior) regions of the brain.

emia and/or hemorrhage), potentially revealing etiologies for perioperative symptoms.

Figure 5 depicts examples in which echo 2 identified lesions with reduced diffusivity that were missed on echo 1 . Here, a site of reduced diffusivity (confirmed on ADC, formerly missed by both echo 1 and the reference DWI) in the contralateral cerebral hemisphere was identified, raising the possibility of an embolic source of infarction.

In 2 cases, echo 2 revealed lesions that would not have been deemed acute (hyperintense lesions on echo 2 DWI but not confirmed by ADC and associated with high signal on FLAIR). In 1 such case, echo 2 revealed a halo of hyperintense signal resembling edema (Fig 6A), prompting the readers to review the ADC map. In another case, echo 2 identified both acute (low signal on ADC) and subacute (isointense to slightly bright signal on ADC) evolving areas of infarction (Fig $6 B$ ) as hyperintense foci. Without the aid of ADC, echo 2 thus overestimated regions of acute ischemia in this case.

Figure 7 shows both echo 1 and echo 2 DWI and ADC of a patient with multiple embolic infarcts. Fourteen new lesions were found on the DWI of echo 2 in this patient, all of which were confirmed as lesions with decreased diffusivity on ADC of echo 1.

\section{Relaxivity Maps}

The $R_{2}$ maps were deemed useful in several regards. Both acute and chronic infarcts demonstrated low $R_{2}$ values that were readily apparent. Even small areas of ischemia were identified on the $\mathrm{R}_{2}$ maps. For instance, $R_{2}$ maps frequently identified lesions presumed to represent chronic small-vessel ischemic disease in the periventricular and subcortical white matter, a finding typically identified on FLAIR and T2-weighted images. The $\mathrm{R}_{2}$ maps identified areas of subarachnoid, old cerebellar, and intraventricular hemorrhage (including some foci that were difficult to visualize on the GRE scan). Mineralization was depicted as regions of high $R_{2}$ and was particularly notable in the basal ganglia (Fig 8).

\section{DISCUSSION}

This work investigated an alternative-approach DWI with the use of a PI-enhanced dual-echo sequence. Typically, DWI sequences use the shortest TE available by the vendor to avoid significant T2-weighting. The primary underlying concern is that a longer TE will result in a lower SNR, and a higher rate of false-positive lesions (that is, lesions that do not have reduced diffusivity) on DWI from increased T2-weighting. However, many lesions with reduced diffusivity also have a prolonged $\mathrm{T} 2$ value compared with the surrounding tissue ${ }^{6,8}$; thus, DWI studies may benefit from a longer TE. Improved conspicuity of ischemic lesions bought by long TEs has also incidentally been observed in a comparison among DWI acquired with different imaging parameters. ${ }^{9}$

The findings in this work support the use of a DWI approach that achieves both a high effective resolution through the use of PI and a long TE to draw the radiologist's attention to acute ischemic lesions. However, because PI effectively shortens the TE, one approach is to "waste" sequence time and use a longer TE, and the other is to incorporate a more sensitive second echo at a reasonable TE, as done in this work, and thereby use the information from both echoes. With the acquisition of 2 echoes acquired at different TEs within 1 diffusion-preparation period, we found that one can use echo 2 for improved sensitivity to ischemic lesions with reduced diffusivity.

\section{Radiologist Ratings}

This study found that the DWI from echo 2 was useful for lesion delineation and improved detection compared with the DWI 


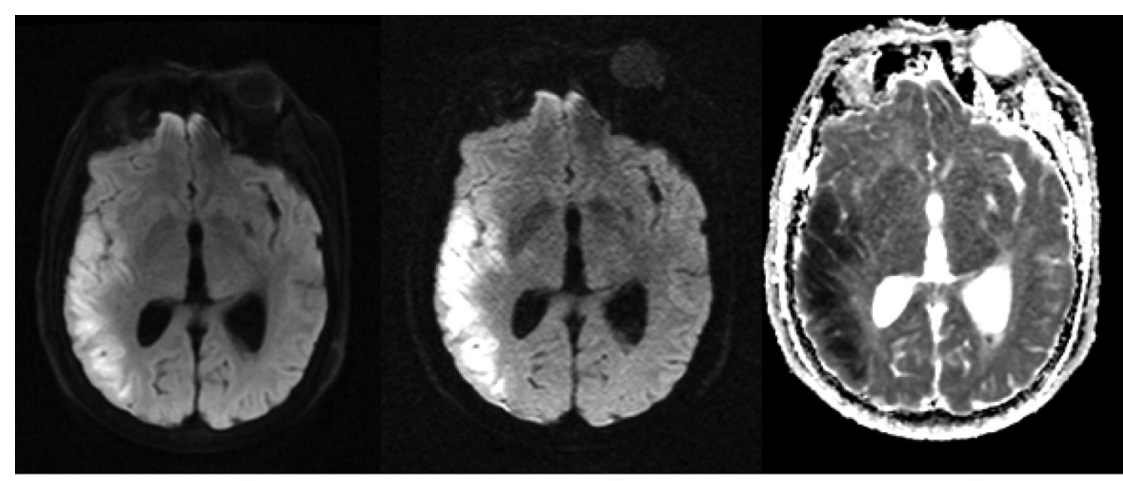

DWl: Echo 1

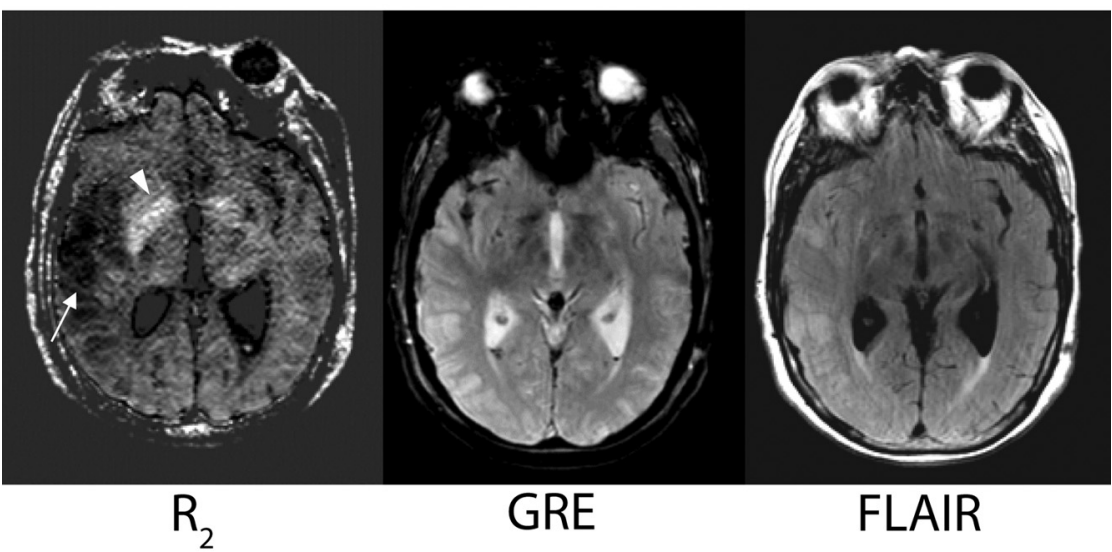

FIG 8. A sample case showing the potential contribution of the $R_{2}$ map. A 69-year-old female patient with vasospasm after subarachnoid hemorrhage. The low- $R_{2}$ lesion is more conspicuous than the corresponding T2 hyperintensity on the FLAIR image. On the basis of DWI/ADC, this area represents acute right MCA territory infarction. The potential contribution of the $R_{2}$ map with regard to timing of stroke and its evolution is unknown but prompts future investigation. The $\mathrm{R}_{2}$ map also shows more conspicuous mineralization in the basal ganglia than the gradientrecalled echo image (arrowhead).

from echo 1 and the product DWI. Echo 2 was rated higher than both echo 1 and the reference product DWI for lesion conspicuity and diagnostic confidence (Fig 2). Echo 1 was rated as better than the product DWI sequence. While several lesions showed higher signal intensity on the product DWI than on echo 1 (related to the intermediate TE of the product DWI [70 ms] between that of echo 1 [48 ms] and that of echo 2 [105 ms]), echo 1 was preferred for its ability to resolve lesions.

Seventy-two additional lesions were identified on echo 2 (that were not seen on echo 1 in the initial reading) in 23 patients diagnosed with acute infarct. Of these, 93\% were deemed lesions with "reduced diffusion" on ADC, $4 \%$ were too small to assess, and the remaining 3\% were chronic lesions. As a result, of the 50 patients scanned for this study, the findings on echo 2 were predicted to have impacted stroke work-up in $16 \%$ of cases ( 8 patients) and potentially influenced $32 \%$ of cases (16 patients).

Echo 2 was rated lower than echo 1 for image quality-presumably on the basis of its reduced SNR. However, our data suggest that the benefit of heightened lesion conspicuity and diagnostic quality of echo 2 far outweighs the resultant decrease in image quality during clinical image interpretation. This benefit becomes particularly evident in the scoring of the 14 studies with negative findings. Despite consistently scoring echo 2 as having lower im- age quality, the clinical confidence in a negative study remained high. We speculate that this reflects the high sensitivity associated with echo 2 . In addition, the more uniform background signal associated with echo 2 allowed greater reliability in radiologic interpretation and in distinguishing infarct from heightened coil sensitivity at the periphery of the image. This observation, coupled with improved sensitivity to lesions with reduced diffusivity, likely increased confidence in interpreting examinations as having negative findings when correlated with subsequent studies.

\section{Representative Cases}

Figure 3 is representative of a case in which the routine product DWI sequence demonstrated lower diagnostic confidence, due to inferior image quality, compared with echoes 1 and 2. In this case, a small infarct present on the dual-echo DWI on Fig $3 C$ was initially missed on the product DWI, a finding that could have prompted further clinical investigation for the source or altered risk for future stroke. Figures 4 and 5 show cases in which echo 2 demonstrated improved lesion conspicuity and diagnostic confidence compared with echo 1. Figure 5 also shows an example of a missed contralateral lesion that would have changed the clinical management of the patient because it raised the possibility of an embolic source of infarction. Note that many lesions detected on echo 2 could be seen retrospectively, either by a side-by-side comparison with echo 1 or the product DWI, or simply by scrolling through the images at a considerably slower rate than is performed in a typical clinical practice.

Of the lesions that were either too small to assess or deemed chronic, we would argue that an image with heightened sensitivity to lesions with a relatively small false-positive rate is preferred over a less sensitive technique where one may miss lesions. Figure 6 shows 2 examples in which the heightened sensitivity of echo 2 to both acute and chronic lesions prompted radiologists to probe other imaging sequences to render the final imaging impression. In these instances, the radiologists still preferred echo 2 because combining the information obtained in echo 2 with the ADC generated from echo 1 improved specificity and guided their diagnostic process. Nevertheless, for any TE, DWI contains mixed contributions from diffusion, proton attenuation, and T2 effects. ${ }^{9}$ Even by using a single-shot EPI technique, T2 contrast on DWI is never completely eliminated because the TE is too long (mainly due to the presence of the diffusion-preparation time). Thus, interpreting echo 2 in isolation can yield false-positive results. DWI should be used to screen for lesions and then should be 
interpreted with reference to images obtained with other sequences, such as FSE, FLAIR, and the ADC map. ${ }^{21}$

\section{Practical Considerations}

While the DWI of echo 2 was considered superior to that of echo 1 in all measures apart from image quality, one must acquire a higher SNR echo 1 image to produce an ADC map to aid diagnostic specificity. Figure 7 shows echo 1 and echo 2 DWI and ADC maps of a patient with multiple embolic infarcts-illustrating the difficulty of decoupling noise from the infarct on the ADC of echo 2 . Figure 7 also demonstrates that echo 2 can be more reliable in distinguishing infarct from heightened coil sensitivity present at the periphery of the image (particularly the posterior brain regions) on echo 1.

\section{Relaxivity Maps}

Another advantage of the dual-echo approach is that $\mathrm{R}_{2}$ maps can be computed from the $b=0$ and $b=1000$ images acquired from the first and second echo, to reveal a potentially useful, additional image contrast typically acquired with a separate scan (Fig 8). Although not thoroughly studied, $\mathrm{R}_{2}$ maps were particularly useful for visualizing hemorrhagic material and hemorrhagic transformation within a stroke, areas of mineralization, and new and old areas of infarction. In this capacity, relaxivity maps generated from the dual-echo process might provide complementary information otherwise obtainable only by requisite sequences such as FLAIR or GRE, though further research will be needed to fully explore this observation.

\section{Limitations}

With a PI factor of 3 to shorten the EPI readout coupled with the clinical DWI parameters used in this work, it was possible to acquire echo 2 with no increase in overall scanning time compared with its single-echo alternative, because its incorporation exploited the dead time of the sequence (ie, for 23 sections, the second echo fit within the TR of 3 seconds that we typically use clinically at our institution). However, a caveat of the dual-echo approach is that the scanning time will increase if one desires thinner sections or a higher spatial resolution than used in this work. For example, a section thickness of $3 \mathrm{~mm}$ coupled with 38 sections to maintain the same brain coverage as used here will result in an $\sim 25 \%$ increase in scanning time compared with the single-echo alternative (for the same in-plane resolution and TR used in this study).

Another limitation of this study is our use of the StejskalTanner diffusion preparation, rather than the twice-refocused $^{19,20}$ diffusion preparation sequence that minimizes eddy current effects. We purposely chose the Stejskal-Tanner approach to help minimize the TE of echo 2-to increase the SNR and avoid excessive T2 contrast; however, this resulted in eddy current-induced artifacts that manifested as blurring in the final DWI of both echoes. These artifacts can be reduced with the use of eddy current-correction methodology. ${ }^{22,23}$

\section{Future Work}

There are additional ways one may be able exploit a dual-echo sequence to reveal useful information. Because both echoes share the same coil sensitivity profile, one can "flatten" the DWI by removing the coil sensitivity. ${ }^{24}$ While one cannot decouple pro- ton attenuation from coil sensitivity by using this approach, the resulting image contrast may be an interesting one to explore and will be the subject of further investigation.

\section{CONCLUSIONS}

The PI-enhanced dual-echo DWI approach is a useful method for evaluating DWI lesions. Echo 2 can be used for added sensitivity in detecting lesions with reduced diffusivity; echo 1, for higher SNR ADC maps, while $\mathrm{R}_{2}$ maps calculated from both echoes may provide a potential source of complementary information. We also demonstrated that many new lesions became apparent on echo 2 that predominantly reflected acute ischemia or brain injury, and we maintain that the TE in DWI can be exploited to draw the radiologist's attention to such lesions.

\section{ACKNOWLEDGMENTS}

We would like to thank Patrick D. Barnes for his generous support and assistance with this study.

Disclosures: Murat Aksoy—RELATED: Grant: National Institutes of Health. Matus Straka-UNRELATED: Employment: Kantonsspital Winterthur, Winterthur Switzerland, OTHER RELATIONSHIPS: Director of Research, iSchemaView Inc. Roland Bammer-RELATED: Grant: National Institute of Biomedical Imaging and Bioengineering, * UNRELATED: Board Membership: iSchemaView Inc, Comment: member, Board of Directors (no payment), Stock/Stock Options: iSchemaView Inc, founder. Michael E Moseley—UNRELATED: Grants/Grants Pending: National Institutes of Health, ${ }^{*} \mathrm{Na}$ tional Institute of Neurological Disorders and Stroke.* Greg Zaharchuk—UNRELATED: Grants/Grants Pending: GE Healthcare.* *Money paid to the institution.

\section{REFERENCES}

1. Le Bihan D, Douek P, Argyropoulou M, et al. Diffusion and perfusion magnetic resonance imaging in brain tumors. Top Magn Reson Imaging 1993;5:25-31

2. Hajnal JV, Doran M, Hall AS, et al. MR imaging of anisotropically restricted diffusion of water in the nervous system: technical, anatomic, and pathologic considerations. J Comput Assist Tomogr 1991;15:1-18

3. Sorensen AG, Buonanno FS, Gonzalez RG, et al. Hyperacute stroke: evaluation with combined multisection diffusion-weighted and hemodynamically weighted echo-planar MR imaging. Radiology 1996;199:391-401

4. Henkelman R. Diffusion-weighted MR imaging: a useful adjunct to clinical diagnosis or a scientific curiosity? AJNR Am J Neuroradiol 1990;11:932-34

5. Patterson DM, Padhani AR, Collins DJ. Technology insight: water diffusion MRI-a potential new biomarker of response to cancer therapy. Nat Clin Pract Oncol 2008;5:220-33

6. Sugahara T, Korogi Y, Kochi M, et al. Usefulness of diffusionweighted MRI with echo-planar technique in the evaluation of cellularity in gliomas. J Magn Reson Imaging 1999;9:53-60

7. Sumi M, Sakihama N, Sumi T, et al. Discrimination of metastatic cervical lymph nodes with diffusion-weighted MR imaging in patients with head and neck cancer. AJNR Am J Neuroradiol 2003;24:1627-34

8. Le Bihan D, Breton E, Lallemand D, et al. MR imaging of intravoxel incoherent motions: application to diffusion and perfusion in neurologic disorders. Radiology 1986;161:401-07

9. Burdette JH, Elster AD, Ricci PE. Acute cerebral infarction: quantification of spin-density and T2 shine-through phenomena on diffusion-weighted MR images. Radiology 1999;212:333-39

10. Pruessmann KP, Weiger M, Scheidegger MB, et al. SENSE: sensitivity encoding for fast MRI. Magn Reson Med 1999;42:952-62

11. Griswold MA, Jakob PM, Heidemann RM, et al. Generalized autocalibrating partially parallel acquisitions (GRAPPA). Magn Reson Med 2002;47:1202-10 
12. Bammer R, Auer M, Keeling SL, et al. Diffusion tensor imaging using single-shot SENSE-EPI. Magn Reson Med 2002;48:128-36

13. Skare S, Newbould RD, Clayton DB, et al. Clinical multishot DWEPI through parallel imaging with considerations of susceptibility, motion, and noise. Magn Reson Med 2007;57:881-90

14. Geijer B, Sundgren PC, Lindgren A, et al. The value of $\mathbf{b}$ required to avoid T2 shine-through from old lacunar infarcts in diffusionweighted imaging. Neuroradiology 2001;43:511-17

15. Feinberg DA, Kiefer B, Litt AW. Dual contrast GRASE (gradientspin echo) imaging using mixed bandwidth. Magn Reson Med 1994;31:461-64

16. Gallichan D, Andersson JL, Jenkinson M, et al. Reducing distortions in diffusion-weighted echo planar imaging with a dual-echo blipreversed sequence. Magn Reson Med 2010;64:382-90

17. Stejskal EO, Tanner JE. Spin diffusion measurements: spin echoes in the presence of a time-dependent field gradient. J Chem Phys 1965;42:288-92

18. Bernstein MA, King KF, Zhou XJ. Handbook of MRI Pulse Sequences. Burlington, Massachusetts: Elsevier Academic Press; 2004
19. Feinberg DA, Jakob PD. Tissue perfusion in humans studied by Fourier velocity distribution, line scan, and echo-planar imaging. Magn Reson Med 1990;16:280-93

20. Reese TG, Heid O, Weisskoff RM, et al. Reduction of eddy-currentinduced distortion in diffusion MRI using a twice-refocused spin echo. Magn Reson Med 2003;49:177-82

21. Beauchamp NJ Jr, Bryan RN. Acute cerebral ischemic infarction: a pathophysiologic review and radiologic perspective. $A J R A m J$ Roentgenol 1978;171:73-84

22. Andersson JL, Skare S, Ashburner J, et al. How to correct susceptibility distortions in spin-echo echo-planar images: application to diffusion tensor imaging. Neuroimage 2003;20:870-88

23. Zeineh MM, Holdsworth $S$, Skare $S$, et al. Ultra-high resolution diffusion tensor imaging of the microscopic pathways of the medial temporal lobe. Neuroimage 2012;62:2065-82

24. Holdsworth SJ, Skare S, Straka M, et al. GRAPPA-accelerated dualecho diffusion-weighted EPI with intensity correction. In: Proceedings of the 20th Annual Meeting of the International Society of Magnetic Resonance in Medicine, Melbourne, Australia. May 5-11, 2012:20 